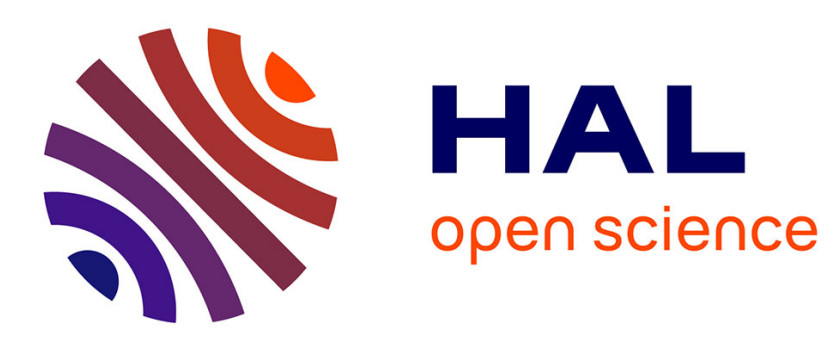

\title{
Adaptive processing of catadioptric images using polarization imaging: toward a polacatadioptric model
}

Samia Ainouz-Zemouche, Olivier Morel, Saleh Mosaddegh, David Fofi, Abdelaziz Bensrhair

\section{- To cite this version:}

Samia Ainouz-Zemouche, Olivier Morel, Saleh Mosaddegh, David Fofi, Abdelaziz Bensrhair. Adaptive processing of catadioptric images using polarization imaging: toward a polacatadioptric model. Optical Engineering, 2013, pp.037001. 10.1117/1.OE.52.3.037001 . hal-00807516

\section{HAL Id: hal-00807516 https://hal.science/hal-00807516}

Submitted on 4 Apr 2013

HAL is a multi-disciplinary open access archive for the deposit and dissemination of scientific research documents, whether they are published or not. The documents may come from teaching and research institutions in France or abroad, or from public or private research centers.
L'archive ouverte pluridisciplinaire HAL, est destinée au dépôt et à la diffusion de documents scientifiques de niveau recherche, publiés ou non, émanant des établissements d'enseignement et de recherche français ou étrangers, des laboratoires publics ou privés. 


\section{Optical Engineering}

\section{Adaptive processing of catadioptric images using polarization imaging: towards a pola-catadioptric model}

Samia Ainouz

Olivier Morel

David Fofi

Saleh Mosaddegh

Abdelaziz Bensrhair 


\section{Adaptive processing of catadioptric images using polarization imaging: towards a pola-catadioptric model}

\author{
Samia Ainouz \\ Institue National des Sciences Appliquées de \\ Rouen \\ Laboratoire d'Informatique du traitement de \\ I'Information et des Systèmes Lab EA4108 \\ 08 Avenue de I'Université, Saint Etienne du \\ Rouvray, 76801, France \\ Email: samia.ainouz@insa-rouen.fr

\section{Olivier Morel \\ David Fofi} \\ Saleh Mosaddegh \\ Burgundy University \\ Laboratoire Electronique Informatique et Images \\ Lab \\ Unité Mixte de Recherche 5158, Institue \\ Universitaire Technologique Le Creusot, 12 rue \\ de la Fonderie, 71200, France
}

\author{
Abdelaziz Bensrhair \\ Institue National des Sciences Appliquées de \\ Rouen \\ Laboratoire d'Informatique du traitement de \\ I'Information et des Systèmes Lab EA4108 \\ 08 Avenue de l'Université, Saint Etienne du \\ Rouvray, 76801, France
}

\begin{abstract}
A nonparametric method to define a pixel neighborhood in catadioptric images is presented. The method is based on an accurate modeling of the mirror shape by mean of polarization imaging. Unlike most processing methods existing in the literature, this method is nonparametric and enables us to respect the catadioptric image's anamorphosis. The neighborhood is directly derived from the two polarization parameters: the angle and the degree of polarization. Regardless of the shape of the catadioptric sensor's mirror (including noncentral configurations), image processing techniques such as image derivation, edge detection, interest point detection, as well as image matching, can be efficiently performed. @ 2013 Society of Photo-Optical Instrumentation Engineers (SPIE) [DOI: 10.1117/1 .OE.52.3.037001]
\end{abstract}

Subject terms: catadioptric images; polarization imaging; adapted neighborhood; processing.

Paper 121547 received Oct. 26, 2012; revised manuscript received Feb. 18, 2013; accepted for publication Feb. 22, 2013; published online Mar. 13, 2013.

\section{Introduction}

Catadioptric sensors are systems consisting of convex mirror and conventional camera [charge coupled device (CCD) or complementary metal-oxide-semiconductor]. ${ }^{1}$ The used mirrors can have several different convex shapes, namely, paraboloidal, spherical, hyperbolical, conical, ellipsoidal, or planar. They produce a hemi-spherical field of view. ${ }^{2}$ Catadioptric devices are widely used in telescopes, teleconferencing systems, virtual reality, monitoring systems, and recently, in the navigation of mobile robots. Images produced by catadioptric sensors contain a significant amount of radial distortion and variation in inherent scale. ${ }^{3}$ Direct application of conventional image processing algorithms without considering an image's anamorphosis yields low performance. This is due to the fact that most of image processing algorithms are based on the pixel's neighborhoods. ${ }^{4,5}$ Edge detection, or image filtering, is carried out by the image convolution with a square window. Interest point detection is mainly based on the convolution of the image and a mask. The correlation function used for the matching is also carried out by a comparison of the pixel's neighborhoods within the images to be matched. These algorithms all need to be adapted for catadioptric images. Indeed, the classical square neighborhood is not suitable for the catadioptric plane topology, where a straight line is imaged as a curve closely defined by the mirror's shape. ${ }^{3}$ Several authors

0091-3286/2013/\$25.00 @ 2013 SPIE have dealt with this problem. Daniilidis ${ }^{3}$ uses a projective space where operators are shift are invariant. The catadioptric image is processed after its projection on a virtual sphere. The final result is obtained by a back projection from the sphere to the image plane. Strauss ${ }^{2,6}$ uses a cylinder as a projective space to perform edge detection and define morphological operators on catadioptric images. The results of these transformations suffer frequently from either a lack of precision because of the intermediate projective spaces or a loss of relevant information, which can decrease the performance of some applications, such as corner detection or image matching. Therefore, a new tool that respects the catadioptric plane anamorphosis is needed. In our last article, ${ }^{4}$ we establish a parametric method to process catadioptric images without any projective plane. This method is mainly based on the parameterization of each point on the mirror. In order to carry out an arbitrary motion in the image, the mirror parameters, after their projection, are considered as the pixel arguments. This process is equivalent to directly projecting a motion from the mirror surface to the image plane. An appropriate window adapted to the catadioptric image topology is consequently defined and used to provide efficient image processing algorithms. This last method gives effective results and handles the problem of losing information. The parameters used to model the mirror point are known after a tedious calibration of the catadioptric sensor. However, the parametric model presents some limitations: the mirror's shape should be known in advance, the single view point constraint (centrality) should be respected, ${ }^{7}$ 
and the optical axes of the camera and mirror (revolution axis) should be perfectly aligned. These conditions are rather complicated to be respected in practice.

In the last decades, thanks to its richness in information, the polarization concept has been strongly explored to analyze, interpret, or process several types of images. ${ }^{8-11}$ This paper aims, by using the polarization imaging, to propose a generic solution for the catadioptric images processing. The proposed method provides an efficient model to define an accurate neighborhood around each pixel within the catadioptric plane. The use of the polarization parametersthe degree and the angle of polarization-enables us to avoid the crucial calibration of the catadioptric sensor and relaxes the three above conditions. Experiments show that the method performs efficiently on catadioptric images (central as well as noncentral configurations).

Images obtained by catadioptric sensors suffer from radial distortions, which make their interpretation and processing complicated. In the case of classical images, each set of image pixels is a regular sampling of part of the threedimensional scene. The processing can be done by a convolution of each pixel with a summative mask, which is generally square. The resulting gray value of the pixel is a combination of that pixel's value and its square neighborhood. The mask is translation invariant and applied in the same way on the whole image.

In the catadioptric plane, a regular grid of the scene is entirely deformed by the convex mirror after projection. A straight line will be transformed into a curve with parameters defined by the mirror shape. Unlike the perspective image, two neighboring pixels on the catadioptric image do not belong any more to the same horizontal or vertical line, but lie on the same curve characterized by the mirror shape. Therefore, the square mask has no sense on the image plane.

\section{Polarization Imaging}

Polarization imaging enables us to study the polarization state of a light wave. ${ }^{12}$ The most common applications in artificial vision are the abilities to distinguish metallic and dielectric objects ${ }^{12}$ and detect transparent surfaces. Polarization imaging gives likewise three-dimensional information of specular objects. ${ }^{7,13}$ The physical principle after being reflected is an unpolarized light wave that becomes partially linearly polarized depending on the normal surface and the refractive index of the media on which it impinges. ${ }^{12}$ A partially linearly polarized light has three parameters: light magnitude $I$, degree of polarization $\rho$, and angle of polarization $\varphi$. In order to measure the polarization parameters, a rotating polarizer $M(\alpha)$ oriented at $\alpha$ is set between the target and the camera (Fig. 1). The element $S_{\text {in }}$ refers to the partially

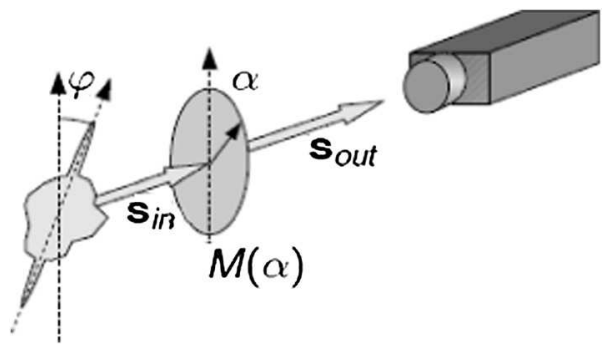

Fig. 1 The polarization device principle. linearly polarized light oriented at $\varphi$, and $S_{\text {out }}$ is the light reaching the camera. To get an automatic system, a liquidcrystal polarization rotator is used instead of the polarizer. It acts as a rotating polarizer, which has the ability to be electrically controlled.

The intensity light $I_{p}$ of each pixel is linked to the polarizer angle $\alpha$ and the polarization parameters by the following equation: ${ }^{7}$

$I_{p}(\alpha)=\frac{I}{2}[\rho \cos (2 \alpha-2 \varphi)+1]$.

The purpose of polarization imaging is to estimate the three parameters $I, \rho$, and $\varphi$ by interpolating the Eq. (1). Because three parameters need to be determined, at least three images, taken with different orientations of the polarizer, are required. The last two parameters, $\rho$ and $\varphi$, are the ones required for defining an adapted neighborhood surrounding each pixel within the catadioptric image.

\section{Catadioptric Images Processing}

Catadioptric vision systems are composed of a CCD camera and a convex mirror. They propose an omnidirectional field of view without any geometrical discontinuities. Figure 2 gives an example of a catadioptric sensor with a parabolic mirror [Fig. 2(a)] and a correspondent catadioptric image [Fig. 2(b)].

In our previous work, ${ }^{4}$ we proposed a parametric model that allows us to process catadioptric images directly on their catadioptric plane. The model respects the image anamorphosis and the nonuniformity of the resolution from the center to the borders of the image. This model works if and only if the above three cited conditions (well calibration, good alignment, and centrality) are satisfied. In practice, these three conditions are hardly satisfied all together. Regarding the acquisition system parameters, the proposed technique is nonparametric. Only the polarization parameters are needed. The three cited conditions are relaxed, and the parameterization of the mirror is not necessary anymore. To better understand the proposed method, the parametric model is first presented.

\subsection{Parametric Model}

The aim of this work is to process catadioptric images directly on their support without any projective space. ${ }^{4}$ The algorithm should also consider the nonuniform resolution of the image after the mirror-plane projection. This problem is widely encountered in the projective methods. ${ }^{3,6}$ To handle these limitations, each point laying on the mirror surface is

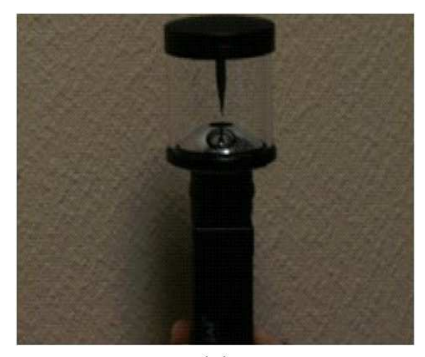

(a)

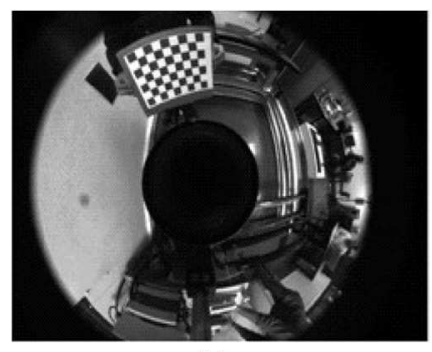

(b)
Fig. 2 (a) Catadioptric sensor; (b) catadioptric image. 
conveniently parameterized. It is important to note that the parameterization is possible if and only if the mirror calibration is well performed. After projection on the image plane, each mirror point's parameters will appear as the arguments of the corresponding pixel. This operation is necessary to carry out a correct motion (rotation, convolution, etc.) within the image. For the parabolic case, for instance, the equation of the parabolic mirror is

$z=-f+\frac{x^{2}+y^{2}}{4 f}$,

where $(x, y, z)$ is a point on the mirror surface, and $f$ is the focal of the mirror, known from calibration. ${ }^{5}$ One possible parameterization of the point $(x, y, z)$ can be given by

$x=f \sqrt{2 t} \cos \tau, \quad y=f \sqrt{2 t} \sin \tau, \quad z=2 f \times(t-1)$,

where $\tau \in[0, \pi]$ and $t \in[0, h]$. Variable $h$ is the distance between the vertex and the boundary of the mirror. Let us now show the impact of this parameterization on the projection from the mirror to the image plane. The general relationship between a point $X=(x, y, z)$ on the mirror and its projection onto the image plane is given by

$\left(\begin{array}{l}u \\ v\end{array}\right)=K_{c} R_{c}\left(X-T_{c}\right)$,

where $(u, v)$ is the projection of the mirror point $X$ onto the image plane, i.e., the pixel coordinates. Variables $K_{c}, R_{c}$ and $T_{c}$, estimated during the calibration, are the intrinsic parameters of the camera, rotation, and translation matrices, respectively, between the mirror coordinates system and the camera system. The translation matrix is defined by the vector $T_{c}=(0,0,-2 e)^{t}$ ( $e$ is the eccentricity of the mirror). $T_{c}$ is the null vector in the case of a parabolic mirror leading to a parallel projection. Replacing $X$ by its arguments $\tau$ and $t$, the corresponding pixel coordinates defined in Eq. (4) will be

$$
\begin{aligned}
& u(t, \tau)=u_{0}+\sigma_{u} \sqrt{2 t} \cos \left(\tau+\frac{\pi}{2}\right) \\
& v(t, \tau)=v_{0}+\sigma_{v} \sqrt{2 t} \sin \left(\tau+\frac{\pi}{2}\right),
\end{aligned}
$$

where $u_{0}, v_{0}, \sigma_{u}, \sigma_{v}$ are the intrinsic parameters of the catadioptric sensor estimated by calibration; ${ }^{14}\left(u_{0}, v_{0}\right)$ is the projection of the camera center to the image plane, and $\left(\sigma_{u}, \sigma_{v}\right)$ is the resolution of the image pixel multiplied by the mirror's focal. In the classical case, an infinitesimal displacement $(h, k)$ in the image is defined by $(u+h, v+k)$. This is erroneous in the case of catadioptric images because the back projection of this motion does not correspond to the true displacement on the mirror surface. Therefore, considering the displacement in the image as the projection of a displacement on the mirror, an infinitesimal motion will be defined by $\{u[t(i+h, j+k), \tau(i+h, j+k)], v[t(i+h, j+k), \tau(i+h, j+k)]\}$, where $i$ and $j$ are the indices of the sampled parameters $t$ and $\tau$. Figure 3 illustrates a projection of a point from the mirror onto the image plane and the shape of its neighborhood.

As a consequence, two neighbors do not lie on the same horizontal or vertical line anymore, but on the same circle of radius $t=\left(u-u_{0} / 2 \sigma_{u}\right)^{2}+\left(v-v_{0} / 2 \sigma_{v}\right)^{2}$ or on the same

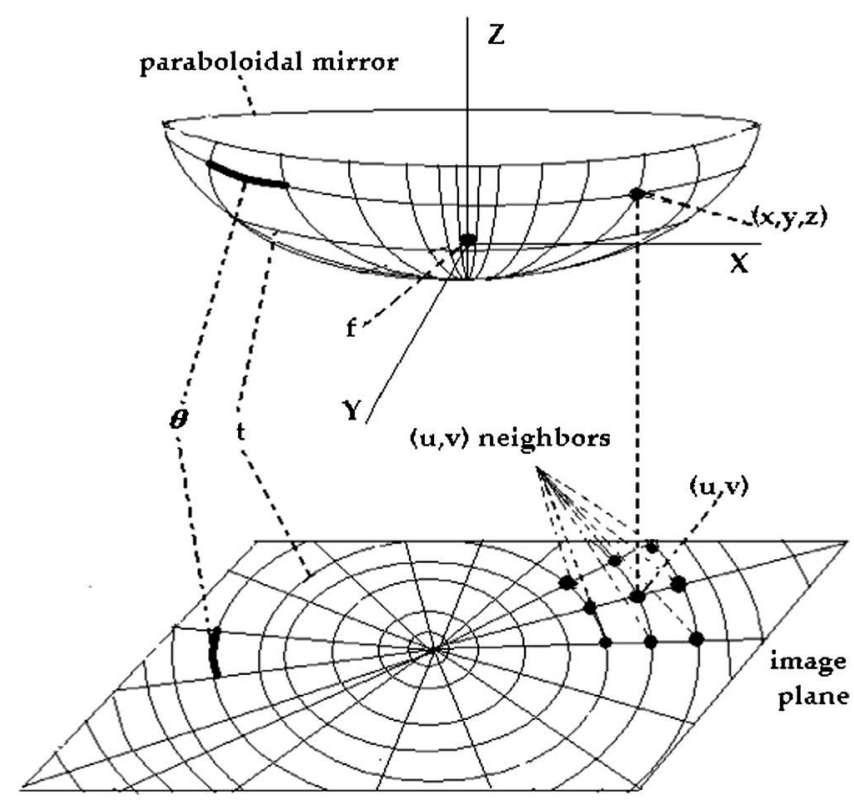

Fig. 3 Sample of the parameterization of a catadioptric image in the orthographic case.

radial of slant $\tau=\operatorname{tg}^{-1}\left[\left(\sigma_{u} / \sigma_{v}\right)\left(v-v_{0} / u-u_{0}\right)\right]$. Figure 4 shows a sample of the projected $t$ and $\tau$ on a real catadioptric image acquired with a parabolic sensor. A great step between circles and radials is chosen to show clearly this projection.

The image convolution is performed with an adapted mask having the same shape of the pixel neighborhood as in Fig. 3. This mask moves in a spiral path from the center of the image into its boundaries following the projected circles and radials. Catadioptric image processing techniques such as image derivative, edge detection, adaptive Harris corner detection and also catadioptric image matching are carried out with this new technique. ${ }^{4}$

\subsection{Nonparametric Case}

Using both Fresnel and Snell-Descartes equations, ${ }^{7,13}$ the normal of each point on the mirror can be deduced from

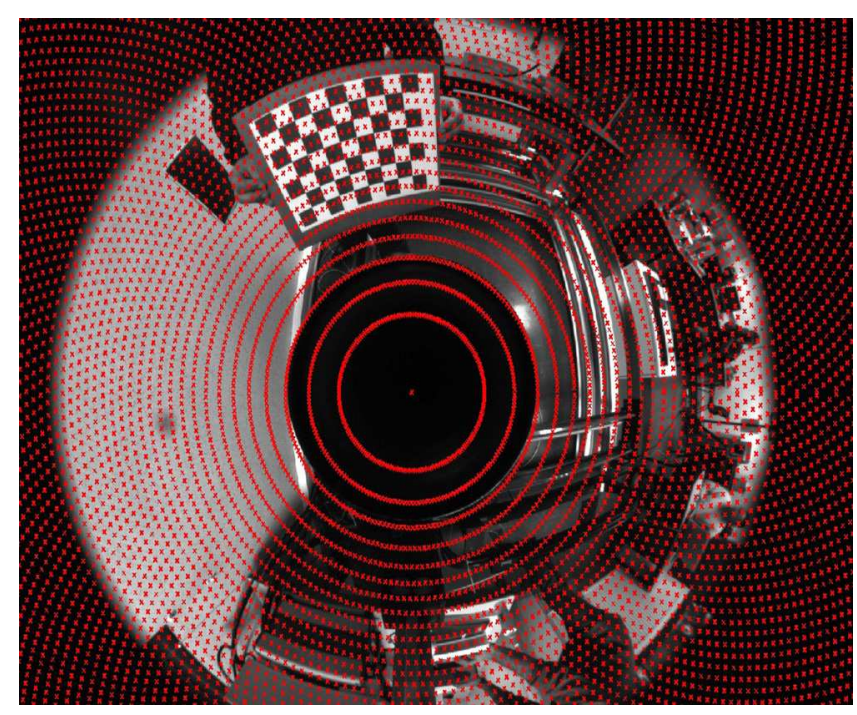

Fig. 4 Example of projection of mirror parameters on catadioptric image plane. 


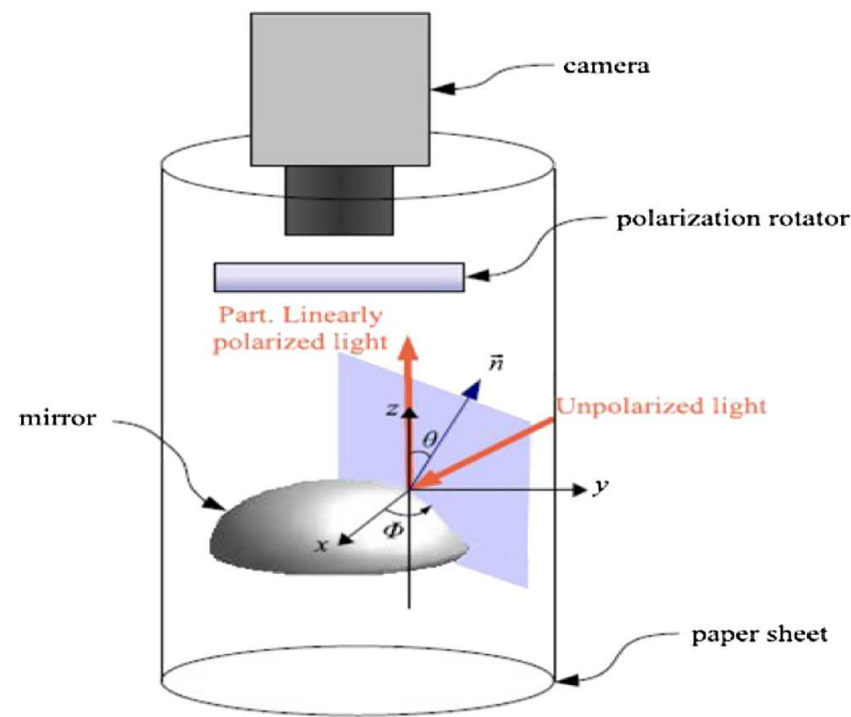

Fig. 5 Catadioptric sensor in a cylindrical sheet of paper.

the polarization parameters. To get an unpolarized light at the incidence, the complete catadioptric system is covered by a cylindrical sheet of paper (Fig. 5).

The normal $\vec{n}$ of the considered point is linked to the azimuthal angle $\theta$ and the zenithal angle $\phi$, with the following formula:

$\vec{n}=\left(\begin{array}{c}\operatorname{tg} \theta \cos \phi \\ \operatorname{tg} \theta \sin \phi \\ 1\end{array}\right)$.

As mentioned above, to measure the polarization parameters related to the mirror of the catadioptric sensor, at least three images are required, one for each rotational angle of the polarizer. Having these images, the system of equations [Eq. (1)] is resolved, and the degree of polarization $\rho$ and angle of polarization $\varphi$ are calculated. ${ }^{7}$ Figure 6 shows the images of the polarization parameters.

The polarization parameters are linked to the normal of each point on the mirror by the Fresnel and Snell Descartes equations: ${ }^{7}$

$\left\{\begin{array}{l}\varphi=\phi \pm \frac{\pi}{2} \\ \rho(\theta)=\frac{2 n t g \theta \sin \theta}{\operatorname{tg}^{2} \theta \sin ^{2} \theta+|\hat{n}|}\end{array}\right.$,

where $\hat{n}$ is the complex refractive index of the surface, up to $0.8+4.5 i$ for a mirror. Based on the investigation of Sturm ${ }^{1}$

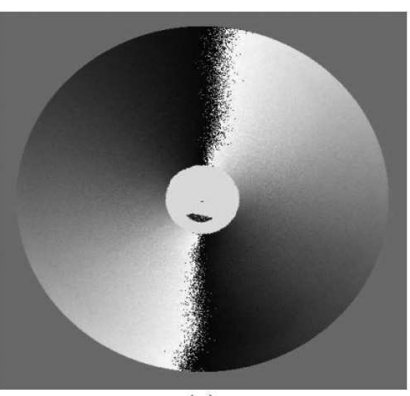

(a)

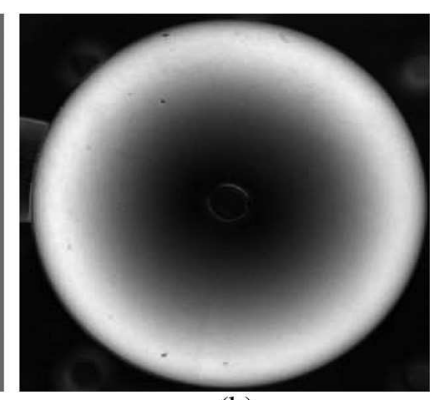

(b)
Fig. 6 (a) Angle of polarization $\varphi$; (b) degree of polarization $\rho$. about the generic concept for the camera calibration, $\mathrm{Morel}^{7}$ uses this relationship to calculate the normal of each point on the mirror to reconstruct the mirror surface. In our case, Eq. (7) is used to define the set of the neighbors of each pixel in the catadioptric image. This new neighborhood allows a convenient processing of the catadioptric image with an appropriate window.

The projection of the angles $\theta$ and $\phi$ on the image plane is the same as the projection of the mirror parameters $t$ and $\tau$. It is shown in Ref. 7, by using the curve of Eq. (7), that the degree of polarization $\rho$ progress in the same way as the angle $\theta$ and the angle of polarization are similar to $\phi$. The equivalence between the polarization parameters and the normal angles allows us to conclude that all the mirror points of the same $\theta$ position have a fixed degree of polarization, and all the mirror points of the same $\phi$ position have a fixed angle of polarization. Finally, from the equivalence between $\theta$ and $\phi$ and between $t$ and $\tau$, two neighbors in the catadioptric image have the same degree of polarization $\rho$ or the same angle of polarization $\varphi$. This conclusion enables us to write the pixel of Eq. (5) as $[u(\rho, \varphi), v(\rho, \varphi)]$ instead of $[u(t, \tau), v(t, \tau)]$. Figure 7(a) shows an example of a set of pixels lying on a fixed $\rho$ (circle) or pixels lying on a fixed $\varphi$ (slant). Figure 7(b) is a zoomed $7 \times 7$-pixel neighborhood of the central pixel in red. As seen in this image, the window is not square, but is adapted to the pixel position and to the resolution of the catadioptric image at that position. With this new window, image processing operators may be introduced as in the parametric method. ${ }^{4}$

\subsection{Image Processing Operators}

In this section, using the proposed theory, image processing operators will be redefined with respect to each application including edge detection, image derivation, interest point detector, and image matching.

\subsubsection{Edge detection}

Consider that $E$ is the set of edges in an arbitrary image $I$ detected by the following formula:

$E(i, j)=\sum_{k} \sum_{l} F(k, l) I(i+k, j+l)$,

where $F$ is an edge detector (Sobel, Prewitt, differential, etc.). In the catadioptric plane, this formula will be

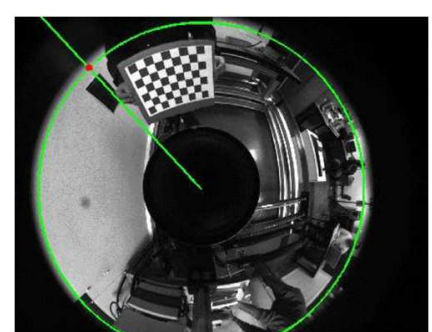

(a)

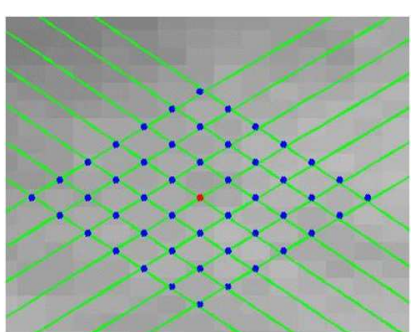

(b)
Fig. 7 (a) Pixel lying on the same degree of polarization (circle) and on the same angle of polarization (slant). (b) A zoomed $7 \times 7$-pixel neighborhood of the central pixel in red. Pixels in blue are the neighbors. 


$$
\begin{aligned}
E(i, j)= & \sum_{k} \sum_{l} F[\rho(k, l), \varphi(k, l)] I[\rho(i+k, j+l), \\
& \varphi(i+k, j+l)] .
\end{aligned}
$$

This formula shows that the displacement within the image is carried out at the $(\rho, \varphi)$ level and not at the pixel level $(u, v)$. The convolution is done with respect to the mask defined in Fig. 7(b). The central value of the edge detector is multiplied with the central pixel of the mask; the other values are also positioned with respect to the mask shape.

\subsubsection{Image derivation}

The second application is the image derivation. Horizontal and vertical derivatives of an image will be circular and radial derivatives in the catadioptric plane. These derivatives are defined by

$$
\begin{aligned}
& \frac{\partial I}{\partial \rho}=\frac{\partial I}{\partial u} \frac{\partial u}{\partial \rho}+\frac{\partial I}{\partial v} \frac{\partial v}{\partial \rho} \\
& \frac{\partial I}{\partial \varphi}=\frac{\partial I}{\partial u} \frac{\partial u}{\partial \varphi}+\frac{\partial I}{\partial v} \frac{\partial v}{\partial \varphi}
\end{aligned}
$$

where $\partial I / \partial u$ refers to the difference between adjacent circles (each one has the fixed degree of polarization), and $\partial I / \partial v$ is the difference of adjacent radials (each one has the fixed angle of polarization). These new derivatives are used to redefine the adapted Harris corner detector. ${ }^{15}$ Usually, for the classical Harris detector, the following matrix $M$ of image derivatives convoluted with a Gaussian kernel $G$ has to be calculated for each pixel:

$M=\left(\begin{array}{cc}\frac{\partial I^{2}}{\partial x} & \frac{\partial I}{\partial x} \frac{\partial I}{\partial y} \\ \frac{\partial I}{\partial x} \frac{\partial I}{\partial y} & \frac{\partial I^{2}}{\partial y}\end{array}\right) \otimes G$

where $\partial I / \partial x$ and $\partial I / \partial y$ are horizontal and vertical derivatives, respectively. Harris corners are pixels for which $R=$ $\operatorname{det}(M)-k \operatorname{trace}(M)$ is positive. Variable $k$ is an empirical value, in general, up to 0.6 . In the catadioptric plane, using the new mask, the matrix $M$ will be written as

$M=\left(\begin{array}{cc}\frac{\partial I^{2}}{\partial \rho} & \frac{\partial I}{\partial \rho} \frac{\partial I}{\partial \varphi} \\ \frac{\partial I}{\partial \rho} \frac{\partial I}{\partial \varphi} & \frac{\partial I^{2}}{\partial \varphi}\end{array}\right) \otimes G$

The multiplication of derivatives as well as the convolution respects the deformed form of Fig. 7(b).

\subsubsection{Image matching}

The last application is the matching of catadioptric images. The matching process needs the measurement of the correlation of pixels lying on the images acquired in different viewpoints. ${ }^{16}$ The correlation function compares the pixel characteristics (gray level, color, texture, etc.) to those of its neighborhood.

The circular and radial neighborhood of each pixel has to be used to evaluate the correlation of each pair of pixels and their neighborhood within different images. For our application, a normalized squared of normal differences (NSSD) function is chosen to measure the dissimilarity between the interest points in the images to be matched. This function is known to be robust to bias and illumination problems between different acquisitions of the same scene. It is defined in case of two acquisitions by

$\operatorname{NSSD}\left(p_{i}, p_{j}\right)=\frac{\left\|\operatorname{neigh}\left(p_{i}\right)-\operatorname{neigh}\left(p_{j}\right)\right\|^{2}}{\left\|\operatorname{neigh}\left(p_{i}\right)\right\|\left\|\operatorname{neigh}\left(p_{j}\right)\right\|}$,

where $p_{i}$ and $p_{j}$ are the pixels lying to the images $i$ and $j$, respectively, to be matched; $\operatorname{neigh}(p)$ is the set of neighbors of pixel $p$. The shape of the mask containing these neighbors is square in the classical case, and has the adapted form in the catadioptric case.

\section{Experiments}

To validate the above-developed theories, experiments with a real catadioptric sensor, central or noncentral, are presented in this section. The experiments include edge detection [Eq. (9)], image derivation [Eq. (10)], interest point detection [Eq. (12)], and image matching [Eq. (13)] for the central case, and image matching in case of noncentral configurations. Experiments are carried out in Fig. 2(b) for the central case.

Usually, the polarimetric acquisitions are spoiled by noise. Pixels having a fixed degree of polarization do not form a perfect circle, and pixels having the same angle of polarization do not form a straight radial. To handle the noise, a mean radius of pixels having the same degree of polarization is calculated, and the resulting circle is considered as the site of the neighbors in the catadioptric image. Similarly, a mean slant of pixels having the same angle of polarization is also considered as the site of neighbors in the image.

\subsection{Adapted Edge Detection}

In order to evaluate the performance of the adapted edge detector, Eqs. (8) (classical) and (9) (adapted) are applied to the catadioptric image of Fig. 2 for $k=l=5$. The results of the edge detection are shown in Fig. 8 for both the classical [Fig. 9(a)] and the adapted [Fig. 9(b)] cases.

The adapted method gives thinner edges that respect the topology of the catadioptric support, whereas the classical edge detection suffers from thick contours and artifacts in the upper circle of the image. This is due to the wrong convolution window surrounding each pixel.

\subsection{Image Derivation}

Circular and radial derivatives are calculated with respect to each pixel using Eq. (10). Figure 8 illustrates the two

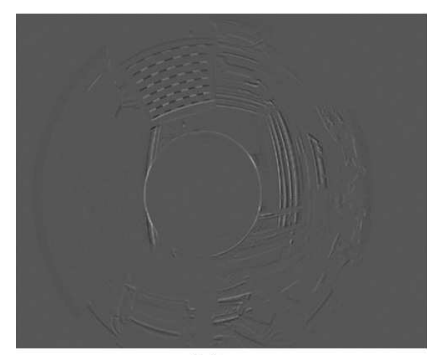

(a)

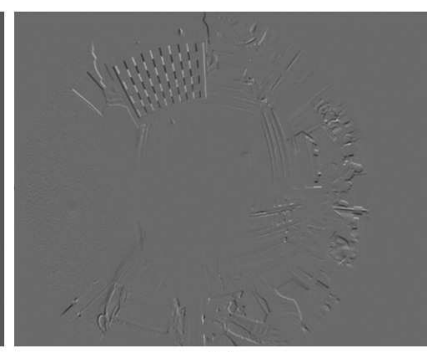

(b)
Fig. 8 (a) Circular derivative; (b) radial derivative. 


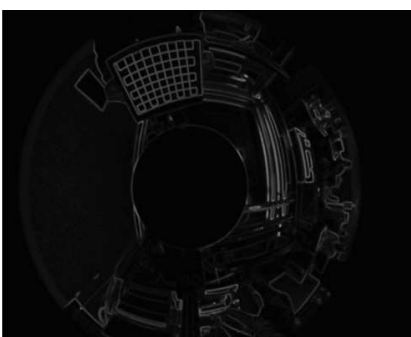

(a)

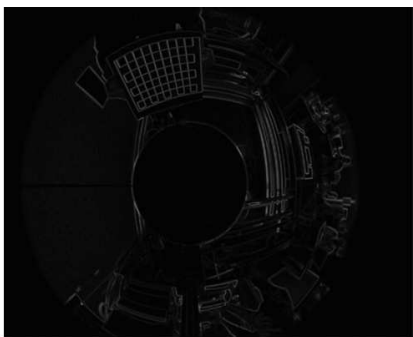

(b)
Fig. 9 (a) Classical edge detection; (b) adapted edge detection.

derivatives. Analyzing the resulted images, one can easily distinguish the circular and radial structures of the image.

\subsection{Adapted Harris Corner Detector}

The comparison between the classical Harris algorithm [Eq. (11)] and the adapted one [Eq. (12)] is illustrated in Fig. 10. The difference is clearly visible. In the adapted case [Fig. 10(b)], more corners are detected compared to the classical case [Fig. 10(a)]. The white circles show the differences. Another difference is the grouping of the detected points. As shown by the violet circles, in the adapted case, the points are more spread out, while they are needlessly grouped in the classical case. This is due to the fact that in the classical case, several corners are erroneously detected because the square neighborhood is badly adapted to the catadioptric plane anamorphosis.

\subsection{Adapted Matching of Catadioptric Imaging}

\subsubsection{Central case}

The NSSD with both the square neighborhood and the adapted one are calculated for each pair of interest points on the image pairs in Fig. 10. If we take the left image as the reference, the motion of the sensor is a rotation of about $80 \mathrm{deg}$. around the optical axis of the camera and a slight translation. Results of the two matching algorithms, classical and adapted, for 20 interest points detected with classical and adapted Harris detectors are illustrated in Figs. 11 and 12, respectively.

In the classical case, all of the interest points are badly matched (green lines) except for one pair of pixels (in blue). The main reason is the already erroneous neighborhood of the pixel. In the new method, however, all the matches are correct. A fixed threshold is imposed to the NSSD, i.e., only $60 \%$ of dissimilarities are taken into account. This makes the algorithm more robust to the outliers.

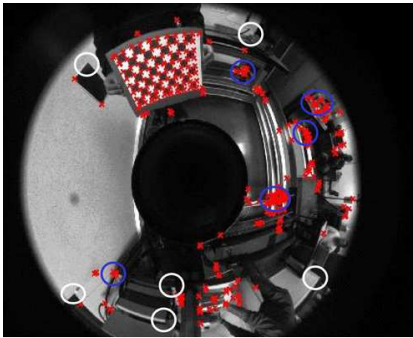

(a)

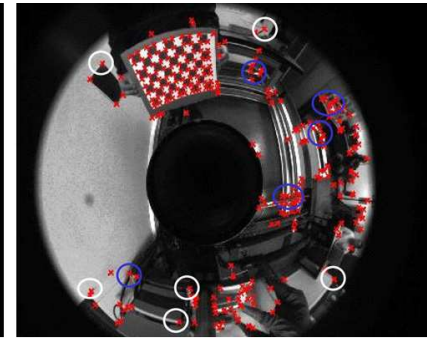

(b)
Fig. 10 Interest points detected by (a) classical Harris corner detector and (b) adapted Harris corner detector.

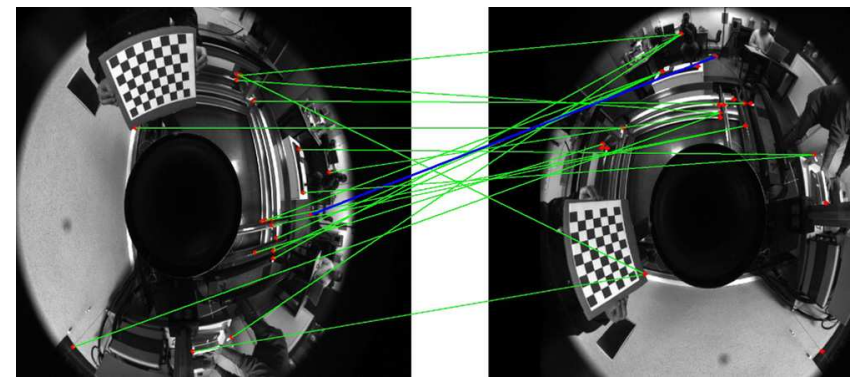

Fig. 11 Classical matching; only one pair of pixels is well matched (in blue).

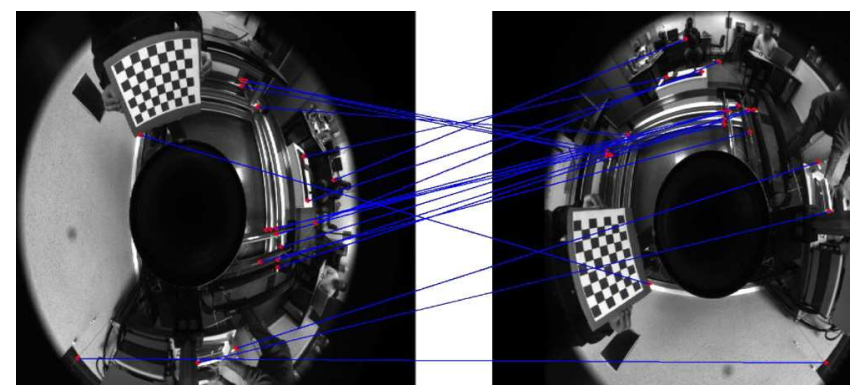

Fig. 12 Adapted matching; all matches are correct.

\subsubsection{Noncentral case}

The second experiment deals with the adapted matching of catadioptric images acquired with noncentral systems. To make the experiment more challenging, the catadioptric sensor is composed of a CCD camera and the back surface of a specular spoon. The algorithm is tested here on a pair of simulated images. The scene is composed from a chair positioned in the corner of a room. The degree of polarization and the angle of polarization images of the spoon are depicted in Fig. 13(a) and 13(b), respectively. There is an arbitrary motion (composed of both translation and rotation) between the positions of the imaging system.

The matching steps (corner detection, image derivation) are entirely carried out with the proposed algorithm. Fifty points of interest are chosen to be matched. The result is illustrated in Fig. 14.

As illustrated, there are two parts of interest points: corners existing in both images and corners without a correspondent in the other image (occlusions). The pairs of pixels apparent in right and left images are correctly matched (in black). The constraint of uniqueness is imposed (one-toone matching) and a fixed threshold is set; pixels for which the NSSD function exceeds $40 \%$ are not matched. One error

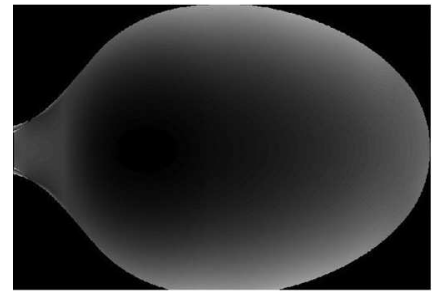

(a)

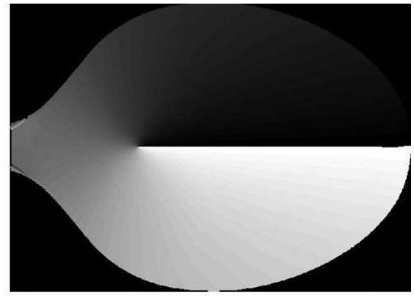

(b)
Fig. 13 (a) Degree of polarization and (b) angle of polarization of the spoon. 


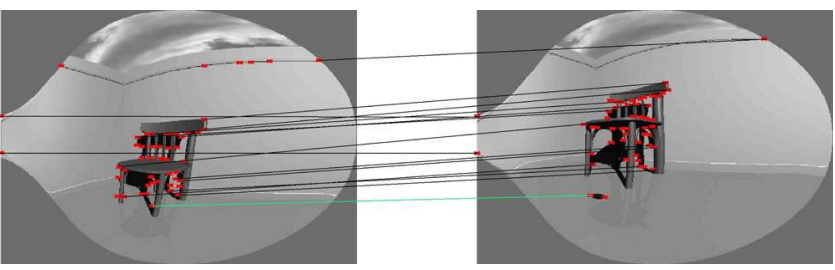

Fig. 14 Adapted matching.

is detected (in green) due to high similarity of the areas surrounding the two pixels.

\section{Conclusion}

An efficient method to process catadioptric images directly on the image plane was presented. This method has the advantage of being nonparametric. Also, the crucial task of calibration is not needed to parameterize the catadioptric sensor. Only the estimation of polarization parameters is sufficient to detect the appropriate neighborhood of each pixel that respects the deformation of the catadioptric image. The algorithm was applied to carry out edge detection, image derivation, interest corners detection, and image matching; the results were interesting when compared to classical processing algorithms.

So far, polarization imaging is being used off-line, as a preparative for image processing and reconstruction. Recent development in the design of polarimetric cameras and the opportunity given now to acquire a powerful and flexible one makes it possible to design a compact catadioptric and polarimetric sensor without adding an external polarizer, filters, or other optical devices. We can now concretely consider the design of an integrated pola-catadioptric camera, which could be easily embedded in various applications. The advantages are manifold; in particular, the opportunity to use polarization imaging on-line and to use it for tasks such as the detection of a (physical) region of interest, target detection, localization, low-level image processing, and so on.

\section{References}

1. P. Sturm and S. Ramalingham, "A generic concept for camera calibration," in Proc. 8th Eur. Conf. Comput. Vis., Lecture Notes in Computer Science, Vol. 3022, pp. 1-13, Springer, Berlin, Heidelberg (2004).

2. O. Strauss and F. Comby, "Fuzzy morphology for omnidirectional images," in Proc. IEEE Int. Conf. on Image Process., Vol. 2, pp. 141-144, IEEE, Genova, Italy (2005)

3. K. Danilidis, A. Makadia, and T. Bülow, "Image processing in catadioptric planes: Spatiotemporal derivatives and optical flow computation," in Proc. IEEE 3rd Workshop on Omnidirectional Vis., pp. 3-10, IEEE, France (2004).

4. S. Ainouz, O. Morel, and D. Fofi, "Mirror-adapted matching of catadioptric images," in Proc. 15th IEEE Int. Conf. on Image Process., pp. 309-312, IEEE, San Diego, California (2008).

5. T. Sbovoda and T. Pajdla, "Epipolar geometry for central catadioptric cameras," Int. J. Comput. Vis. 49(1), 23-37 (2002).

6. F. Jacquey, F. Comby, and O. Strauss, "Fuzzy edge detection for omnidirectional images," Fuzzy Sets Syst. 159(15), 1991-2010 (2008).

7. O. Morel and D. Fofi, "Calibration of catadioptric sensors by polarization images," in Proc. IEEE Int. Conf. on Robotics and Automation, pp. 3939-3944, IEEE, Roma (2007).

8. S. Ainouz, et al., "Physical interpretation of polarization encoded image by color preview," Opt. Exp. 14(13), 5916-5927 (2006).

9. A. Bénière et al., "Precision of degree of polarization estimation in the presence of additive Gaussian detector," Opt. Сommun. 278(2), 264-269 (2007)

10. P. Miché, A. Bensrhair, and D. Lebrun, "Passive 3-D shape recovery of unknown objects using cooperative polarimetric and radiometric stereo vision processes," Opt. Eng. 44(2), 027005 (2005).

11. I. Ibrahim, Pet al., "Illumination invariance and shadow compensation via spectro-polarimetry technique," Opt. Eng. 51(10), 107004 (2012).
12. L. B. Wolff, "Polarization-based material classification from specular reflection," IEEE Trans. Pattern Anal. Mach. Intell. 12(11), 10591071 (1990).

13. L. B. Wolff and T. E. Boult, "Constraining objects feature using a polarization reflectance model," IEEE Trans. Pattern Anal. Mach. Intell. 13(7), 635-657 (1991).

14. C. Mei and P. Rives, "Non biased calibration of a catadioptric central sensor," in Reconnaissance de Formes et Intelligence Artificielle, France (2006).

15. C. Harris and M. Stephens, "A combine corner and edge detector," in Proc. of 4th Alvey Vis. Conf., Manchester, United Kingdom, pp. $147-151$ (1988).

16. S. Chambon and A. Crouzil, "Towards correlation-based matching algorithms that are robust near occlusions," in Proc. 17th Int. Conf. on Pattern Recognit., Vol. 3, pp. 20-23, IEEE, Cambridge (2004).

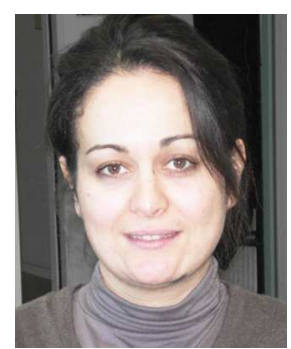

Samia Ainouz received her $\mathrm{PhD}$ degree in image processing from Louis Pasteur University, Strasbourg. She carried out her postdoctoral work in 3D vision at Le2i UMR 6306 CNRS Lab. Since September 2008, she has worked as an associate professor in the LITIS Lab with the Intelligent Transportation Systems Team. Her main research interests are polarization imaging, stereovision, catadioptric vision, and applications of these technics to intelligent vehicles.

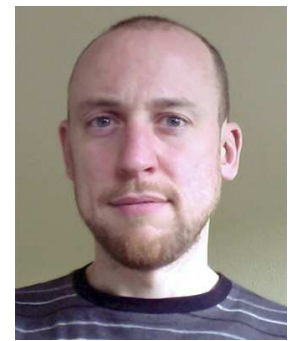

Olivier Morel received his engineering degree in automatic and industrial computing systems from Université de Savoie (Polytech'Savoie), in 2001. He graduated from University of Burgundy in 2002 with a MSc degree in computer vision and image processing. In November 2005, he received the $\mathrm{PhD}$ degree in computer vision from the University of Burgundy. Since September 2007, he has worked as an associate professor in the vision department of the Le2i UMR 6306 CNRS Lab. His main research interests are polarization imaging, $3 \mathrm{D}$ vision, catadioptric vision, and applications of these technics to robotics.

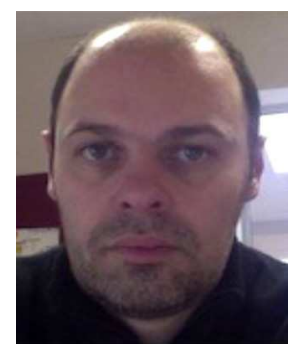

David Fofi is currently a professor at the University of Burgundy, head of the computer vision department of the Le2i UMR CNRS 6306 and coordinator of the Erasmus Mundus Masters in Vision and Robotics (VIBOT). $\mathrm{He}$ received an MSc degree in image and signal processing of the University of CergyPontoise/ENSEA in 1997, and a PhD in computer vision from the University of Picardie Jules Verne, in 2001. He has been awarded a research fellowship from the SnT (University of Luxembourg) since 2012. His research interest includes multiple-view geometry, catadioptric vision, projector-camera systems, and structured light. He participated in and led several French and European projects in the field of computer vision (Erasmus Mundus, CNRS, ANR, PHC, etc.). Since 1998, he has published more than 20 papers in international peer-reviewed journals, 2 patents, and more than 50 conference papers.

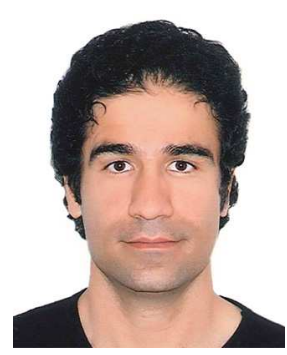

Saleh Mosaddegh received his first MSc degree in industrial engineering from Isfahan University of Technology, Iran, in 2003 and his second MSc degree in computer science (computer vision, Vibot program, a joint European master of science in Vision and Robotics) in Heriot Watt University, United Kingdom, Universitat de Girona, Spain and University of Bourgogne, France in 2008. He was a lecturer at the Industrial Engineering Department, Isfahan University of Technology, Iran, from 2004 to 2006. In September 2011, he received his $\mathrm{PhD}$ degree in robotics and computer vision from the University of Burgundy. He is currently a researcher in Greyc Lab, University of Caen. His main research interests are 3D vision, structure from motion, catadioptric vision, human machine interface, and augmented/mixed reality. 


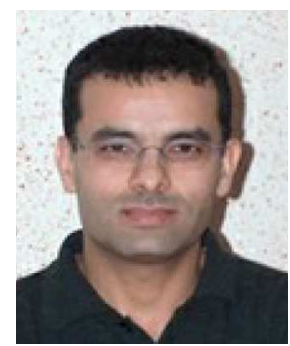

Abdelaziz Bensrhair graduated with an MSc in electrical engineering (1989) and a PhD degree in computer science (1992) at the University of Rouen, France. From 1992 to 1999, he was an assistant professor in the Physic and Instrumentation Department, University of Rouen. He is currently a professor in information systems architecture department, head of Intelligent Transportation Systems Division and co-director of the Computer Science, Information Processing, and Systems Laboratory (LITIS) of the National Institute of Applied Science Rouen (INSAR). 